\title{
Synchronised firing patterns in a random network of adaptive exponential integrate-and-fire neuron model
}

\author{
F. S. Borges ${ }^{1}$, P. R. Protachevicz ${ }^{2}$, E. L. Lameu ${ }^{2}$, R. C. Bonetti ${ }^{2}$, K. C. $\operatorname{Iarosz}^{1,3, *}$, I. L. Caldas ${ }^{1}$, M. S. Baptista ${ }^{3}$, A. \\ M. Batista ${ }^{1,2,3,4, *}$ \\ ${ }^{1}$ Instituto de Física, Universidade de São Paulo, São Paulo, SP, Brazil. \\ ${ }^{2}$ Pós-Graduação em Ciências/Física, Universidade Estadual de Ponta Grossa, Ponta Grossa, PR, Brazil. \\ ${ }^{3}$ Institute for Complex Systems and Mathematical Biology, Aberdeen, SUPA, UK. \\ ${ }^{4}$ Departamento de Matemática e Estatística, Universidade Estadual de Ponta Grossa, Ponta Grossa, PR, Brazil.
}

\begin{abstract}
We have studied neuronal synchronisation in a random network of adaptive exponential integrate-and-fire neurons. We study how spiking or bursting synchronous behaviour appears as a function of the coupling strength and the probability of connections, by constructing parameter spaces that identify these synchronous behaviours from measurements of the inter-spike interval and the calculation of the order parameter. Moreover, we verify the robustness of synchronisation by applying an external perturbation to each neuron. The simulations show that bursting synchronisation is more robust than spike synchronisation.
\end{abstract}

Keywords: synchronisation, integrate-and-fire, network PACS: 05.45.Ra, 87.19.lj

\section{Introduction}

The concept of synchronisation is based on the adjustment of rhythms of oscillating systems due to their interaction (Pikovsky et al., 2001). Synchronisation phenomenon was recognised by Huygens in the 17th century, time when he performed experiments to understand this phenomenon (Bennet et al., 2002). To date, several kinds of synchronisation among coupled systems were reported, such as complete (Li \& Li, 2016), phase (Pereira et al., 2007; Batista et al., 2010), lag (Huang et al., 2014), and collective almost synchronisation (Baptista et al., 2012).

Neuronal synchronous rhythms have been observed in a wide range of researches about cognitive functions (Wang, 2010; Hutcheon \& Yarom, 2000). Electroencephalography and magnetoencephalography studies have been suggested that neuronal synchronisation in the gamma frequency plays a functional role for memories in humans (Axmacher et al., 2006; Fell \& Axmacher, 2011). Steinmetz et al. (Steinmetz et al., 2000) investigated the synchronous behaviour of pairs of neurons in the secondary somatosensory cortex of monkey. They found that attention modulates oscillatory neuronal synchronisation in the somatosensory cortex. Moreover, in the literature it has been proposed that there is a relationship between con-

\footnotetext{
*Corresponding author: antoniomarcosbatista@gmail.com; kiarosz@gmail.com; fernandodasilvaborges@gmail.com
}

scious perception and synchronisation of neuronal activity (Hipp et al., 2011).

We study spiking and bursting synchronisation between neuron in a neuronal network model. A spike refers to the action potential generated by a neuron that rapidly rises and falls (de Lange \& Hasler, 2008), while bursting refers to a sequence of spikes that are followed by a quiescent time (Wu et al., 2012). It was demonstrated that spiking synchronisation is relevant to olfactory bulb (Davison et al., 2001) and is involved in motor cortical functions (Riehle et al., 1997). The characteristics and mechanisms of bursting synchronisation were studied in cultured cortical neurons by means of planar electrode array (Maeda et al., 1995). Jefferys \& Haas (Jefferys \& Haas, 1982) discovered synchronised bursting of CA1 hippocampal pyramidal cells.

There is a wide range of mathematical models used to describe neuronal activity, such as the cellular automaton (Viana et al., 2014), the Rulkov map (Rulkov, 2001), and differential equations (Hodgkin \& Huxley, 1952; Hindmarsh \& Rose, 1984). One of the simplest mathematical models and that is widely used to depict neuronal behaviour is the integrate-and-fire (Lapicque, 1907), which is governed by a linear differential equation. A more realistic version of it is the adaptive exponential integrate-and-fire (aEIF) model which we consider in this work as the local neuronal activity of neurons in the network. The aEIF is a two-dimensional integrate-and-fire model introduced by 
Brette \& Gerstner (Brette \& Gerstner, 2005). This model has an exponential spike mechanism with an adaptation current. Touboul \& Brette (Touboul \& Brette, 2008) studied the bifurcation diagram of the aEIF. They showed the existence of the Andronov-Hopf bifurcation and saddlenode bifurcations. The aEIF model can generate multiple firing patterns depending on the parameter and which fit experimental data from cortical neurons under current stimulation (Naud et al., 2008).

In this work, we focus on the synchronisation phenomenon in a randomly connected network. This kind of network, also called Erdös-Rényi network (Erdös \& Rényi, 1959), has nodes where each pair is connected according to a probability. The random neuronal network was utilised to study oscillations in cortico-thalamic circuits (Gelenbe \& Cramer, 1998) and dynamics of network with synaptic depression (Senn et al., 1996). We built a random neuronal network with unidirectional connections that represent chemical synapses.

We show that there are clearly separated ranges of parameters that lead to spiking or bursting synchronisation. In addition, we analyse the robustness to external perturbation of the synchronisation. We verify that bursting synchronisation is more robustness than spiking synchronisation. However, bursting synchronisation requires larger chemical synaptic strengths, and larger voltage potential relaxation reset to appear than those required for spiking synchronisation.

This paper is organised as follows: in Section II we present the adaptive exponential integrate-and-fire model. In Section III, we introduce the neuronal network with random features. In Section IV, we analyse the behaviour of spiking and bursting synchronisation. In the last Section, we draw our conclusions.

\section{Adaptive exponential integrate-and-fire}

As a local dynamics of the neuronal network, we consider the adaptive exponential integrate-and-fire (aEIF) model that consists of a system of two differential equations (Brette \& Gerstner, 2005) given by

$$
\begin{aligned}
C \frac{d V}{d t}= & -g_{L}\left(V-E_{L}\right)+g_{L} \Delta_{T} \exp \left(\frac{V-V_{T}}{\Delta_{T}}\right) \\
& +I-w \\
\tau_{w} \frac{d w}{d t}= & a\left(V-E_{L}\right)-w
\end{aligned}
$$

where $V(t)$ is the membrane potential when a current $I(t)$ is injected, $C$ is the membrane capacitance, $g_{L}$ is the leak conductance, $E_{L}$ is the resting potential, $\Delta_{T}$ is the slope factor, $V_{T}$ is the threshold potential, $w$ is an adaptation variable, $\tau_{w}$ is the time constant, and $a$ is the level of subthreshold adaptation. The parameter $\Delta_{T}$ controls the sharpness of the initial phase of the spike (Badel et al., 2008) and in the limit $\Delta_{T} \rightarrow 0$ the neuron model becomes a standard leaky integrate-and-fire neuron model (Clopath et al., 2007). If $V(t)$ reaches the threshold $V_{\text {peak }}$, a reset condition is applied: $V \rightarrow V_{r}$ and $w \rightarrow w_{r}=w+b$. In our simulations, we consider $C=200.0 \mathrm{pF}, g_{L}=12.0 \mathrm{nS}, E_{L}=$ $-70.0 \mathrm{mV}, \Delta_{T}=2.0 \mathrm{mV}, V_{T}=-50.0 \mathrm{mV}, I=509.7 \mathrm{pA}$, $\tau_{w}=300.0 \mathrm{~ms}, a=2.0 \mathrm{nS}$, and $V_{\text {peak }}=20.0 \mathrm{mV}$ (Naud et al., 2008).

The firing pattern depends on the reset parameters $V_{r}$ and $b$. Table 1 exhibits some values that generate five different firing patterns (Fig. 1). In Fig. 1 we represent each firing pattern with a different colour in the parameter space $b \times V_{r}$ : adaptation in red, tonic spiking in blue, initial bursting in green, regular bursting in yellow, and irregular in black. In Figs. 1(a), 1(b), and 1(c) we observe adaptation, tonic spiking, and initial burst pattern, respectively, due to a step current stimulation. Adaptation pattern has increasing inter-spike interval during a sustained stimulus, tonic spiking pattern is the simplest regular discharge of the action potential, and the initial bursting pattern starts with a group of spikes presenting a frequency larger than the steady state frequency. The membrane potential evolution with regular bursting is showed in Fig. 1(d), while Fig. 1(e) displays irregular pattern.

Table 1: Reset parameters.

\begin{tabular}{ccccc}
\hline Firing patterns & Fig. & $\mathrm{b}(\mathrm{pA})$ & $V_{r}(\mathrm{mV})$ & Layout \\
\hline adaptation & $1(\mathrm{a})$ & 60.0 & -68.0 & red \\
tonic spiking & $1(\mathrm{~b})$ & 5.0 & -65.0 & blue \\
initial burst & $1(\mathrm{c})$ & 35.0 & -48.8 & green \\
regular bursting & $1(\mathrm{~d})$ & 40.0 & -45.0 & yellow \\
irregular & $1(\mathrm{e})$ & 41.2 & -47.4 & black \\
\hline
\end{tabular}
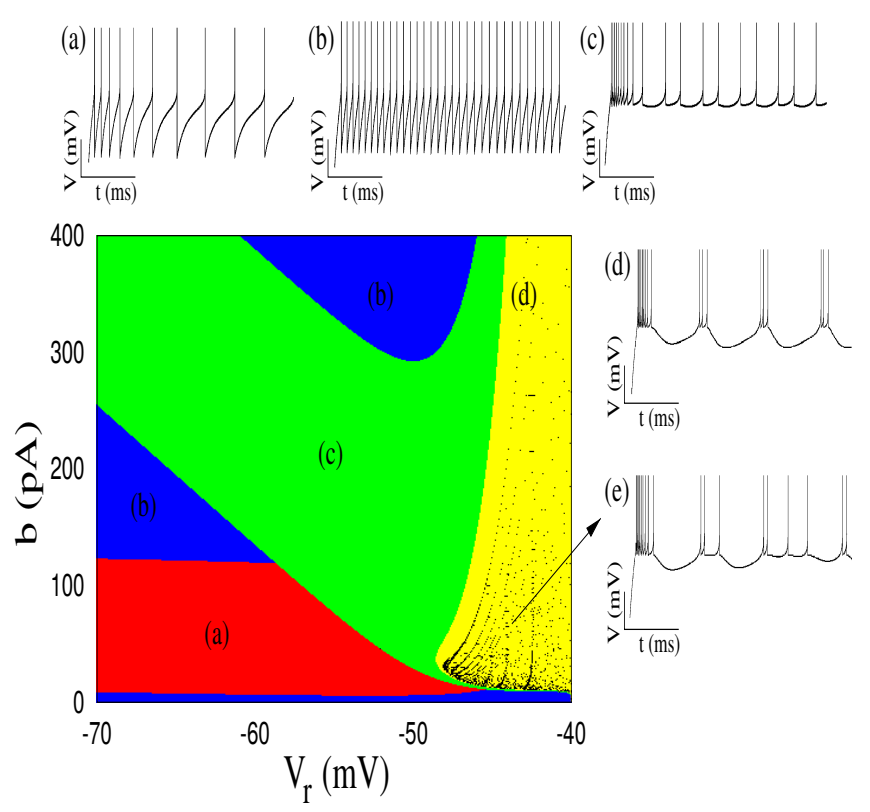

Figure 1: (Colour online) Parameter space for the firing patterns as a function of the reset parameters $V_{r}$ and $b$. (a) Adaptation in red, (b) tonic spiking in blue, (c) initial bursting in green, (d) regular bursting in yellow, and (e) irregular in black. 
As we have interest in spiking and bursting synchronisation, we separate the parameter space into a region with spike and another with bursting patterns (Fig. 2). To identify these two regions of interest, we use the coefficient of variation (CV) of the neuronal inter-spike interval (ISI), that is given by

$$
\mathrm{CV}=\frac{\sigma_{\text {ISI }}}{\overline{\mathrm{ISI}}}
$$

where $\sigma_{\text {ISI }}$ is the standard deviation of the ISI normalised by the mean ISI (Gabbiani \& Koch, 1998). Spiking patterns produce $\mathrm{CV}<0.5$. Parameter regions that represent the neurons firing with spiking pattern are denoted by gray colour in Fig. 2. Whereas, the black region represents the bursting patterns, which results in $\mathrm{CV} \geq 0.5$.

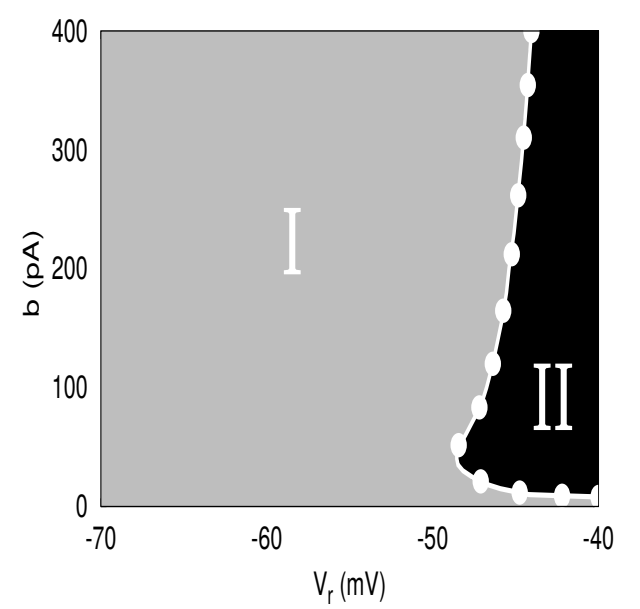

Figure 2: Parameter space for the firing patterns as a function of the reset parameters $V_{r}$ and $b$. Spike pattern in region I $(\mathrm{CV}<0.5)$ and bursting pattern in region II $(\mathrm{CV} \geq 0.5)$ are separated by white circles.

\section{Spiking or bursting synchronisation}

In this work, we constructed a network where the neurons are randomly connected (Erdös \& Rényi, 1959). Our network is given by

$$
\begin{aligned}
C \frac{d V_{i}}{d t}= & -g_{L}\left(V_{i}-E_{L}\right)+g_{L} \Delta_{T} \exp \left(\frac{V_{i}-V_{T}}{\Delta_{T}}\right) \\
& +I_{i}-w_{i}+g_{\mathrm{s}}\left(V_{\mathrm{REV}}-V_{i}\right) \sum_{j=1}^{N} A_{i j} s_{j} \\
& +\Gamma_{i}, \\
\tau_{w} \frac{d w_{i}}{d t}= & a_{i}\left(V_{i}-E_{L}\right)-w_{i}, \\
\tau_{\mathrm{s}} \frac{d s_{i}}{d t}= & -s_{i} .
\end{aligned}
$$

where $V_{i}$ is the membrane potential of the neuron $i, g_{\mathrm{s}}$ is the synaptic conductance, $V_{\mathrm{REV}}$ is the synaptic reversal potential, $\tau_{\mathrm{s}}$ is the synaptic time constant, $s_{i}$ is the synaptic weight, $A_{i j}$ is the adjacency matrix, $\Gamma_{i}$ is the external perturbation, and $a_{i}$ is randomly distributed in the interval $[1.9,2.1]$ to simulate a network composed by neurons with different parameters. We consider $I_{i}=509.7 \mathrm{pA}$, $V_{\mathrm{REV}}=0 \mathrm{mV}$ (excitatory) or $V_{\mathrm{REV}}=-80 \mathrm{mV}$ (inhibitory), $\tau_{\mathrm{s}}=2.728 \mathrm{~ms}$, and $N=100$. For this $I_{i}$ value, when the neurons are uncoupled $\left(g_{\mathrm{s}}=0\right)$, they spike with a frequency of about $9.2 \mathrm{~Hz}$. In addition, $I_{i}$ is a control parameter that can avoid large firing rate. The synaptic conductance $g_{\mathrm{s}}$ represent the strength of the excitatory $\left(g_{\mathrm{ex}}\right)$ or inhibitory $\left(g_{\text {in }}\right)$ connections. The time-step of integration is $10^{-2}$.

We consider a neuronal network model where each neuron is randomly linked to other neurons with a probability $p$ by means of directed connections. When $p$ is equal to 1 , the neuronal network becomes an all-to-all network. A network with this topology was used by Borges et al. (Borges et al., 2016) to study the effects of the spike timing-dependent plasticity on the synchronisation in a Hodgkin-Huxley neuronal network.

A useful diagnostic tool to determine synchronous behaviour is the complex phase order parameter defined as (Kuramoto, 2003)

$$
z(t)=R(t) \exp (\mathrm{i} \Phi(t)) \equiv \frac{1}{N} \sum_{j=1}^{N} \exp \left(\mathrm{i} \psi_{j}\right),
$$

where $R$ and $\Phi$ are the amplitude and angle of a centroid phase vector, respectively, and the phase is given by

$$
\psi_{j}(t)=2 \pi m+2 \pi \frac{t-t_{j, m}}{t_{j, m+1}-t_{j, m}},
$$

where $t_{j, m}$ corresponds to the time when a spike $m(m=$ $0,1,2, \ldots)$ of a neuron $j$ happens $\left(t_{j, m}<t<t_{j, m+1}\right)$. We have considered the beginning of the spike when $V_{j}>$ $-20 \mathrm{mV}$. The value of the order parameter magnitude goes to 1 in a totally synchronised state. To study the neuronal synchronisation of the network, we have calculated the time-average order-parameter, that is given by

$$
\bar{R}=\frac{1}{t_{\text {fin }}-t_{\text {ini }}} \sum_{t_{\text {ini }}}^{t_{\text {fin }}} R(t),
$$

where $t_{\text {fin }}-t_{\text {ini }}$ is the time window for calculating $\bar{R}$. We consider a time window equal to $10^{4} \mathrm{~ms}$ and one trial to compute $\bar{R}$ due to the fact that the results do not have any qualitative dependence on the initial conditions.

Figures 3(a), 3(b), and 3(c) show the raster plots of the excitatory neuronal network for $g_{\mathrm{ex}}=0.02 \mathrm{nS}, g_{\mathrm{ex}}=$ $0.19 \mathrm{nS}$, and $g_{\mathrm{ex}}=0.45 \mathrm{nS}$, respectively, considering $V_{r}=$ $-58 \mathrm{mV}, p=0.5$, and $b=70 \mathrm{pA}$, where the dots correspond to the spiking activities generated by neurons. For $g_{\mathrm{ex}}=0.02 \mathrm{nS}$ (Fig. 3(a)) the network displays a desynchronised state, and as a result, the order parameter values are very small (black line in Fig. 3(d)). Increasing the synaptic conductance for $g_{\mathrm{ex}}=0.19 \mathrm{nS}$, the neuronal network exhibits spike synchronisation (Fig. 3(b)) and the order parameter values are near unity (red line in Fig. 3(d)). When the network presents bursting synchronisation (Fig. 
$3(\mathrm{c})$ ), the order parameter values vary between $R \approx 1$ and $R \ll 1$ (blue line in Fig. $3(\mathrm{~d}))$. $R \ll 1$ to the time when the neuron are firing.
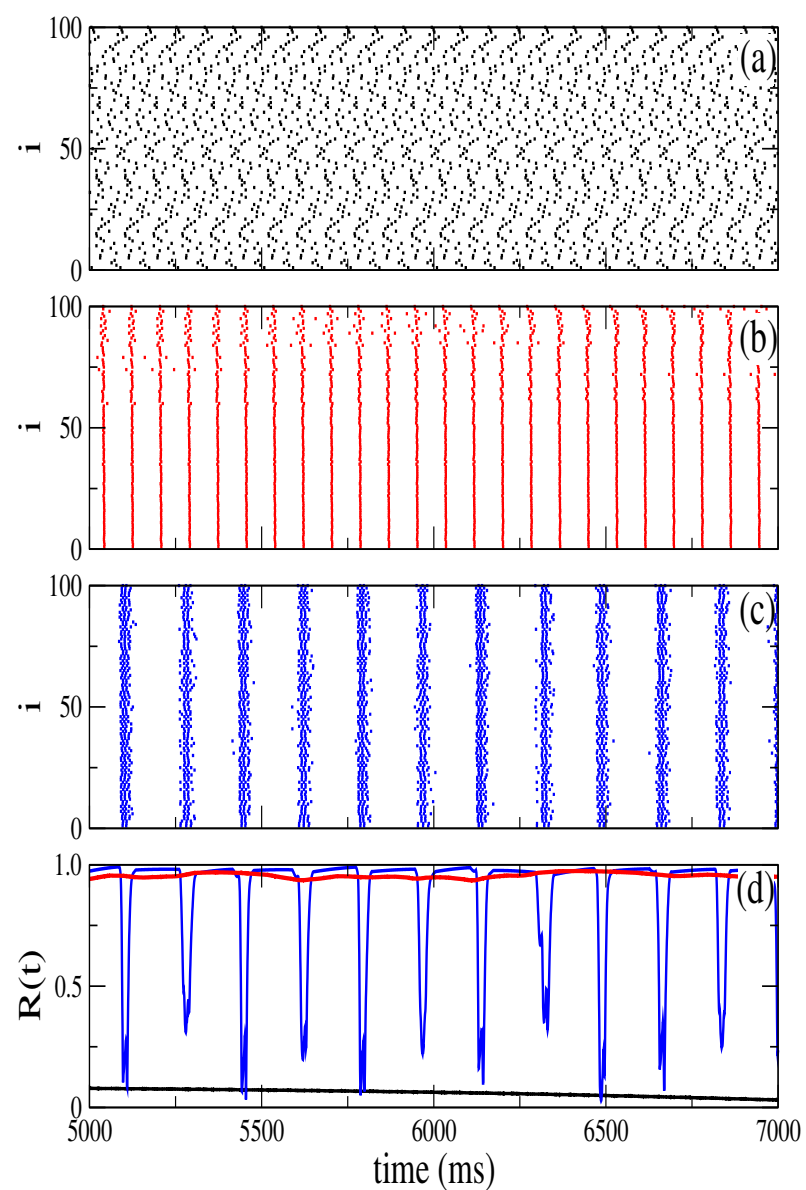

Figure 3: (Colour online) Raster plot of the excitatory neuronal network for (a) $g_{\mathrm{ex}}=0.02 \mathrm{nS}$, (b) $g_{\mathrm{ex}}=0.19 \mathrm{nS}$, and (c) $g_{\mathrm{ex}}=0.45 \mathrm{nS}$, considering $V_{r}=-58 \mathrm{mV}, p=0.5$, and $b=70 \mathrm{pA}$. In (d) the order parameter is computed for $g_{\mathrm{ex}}=0.02 \mathrm{nS}$ (black line), $g_{\mathrm{ex}}=0.19 \mathrm{nS}$ (red line), and $g_{\mathrm{ex}}=0.45 \mathrm{nS}$ (blue line).

In Fig. 4(a) we show $\bar{R}$ as a function of $g_{\text {ex }}$ of the excitatory neuronal network for $p=0.5, b=50 \mathrm{pA}$ (black line), $b=60 \mathrm{pA}$ (red line), and $b=70 \mathrm{pA}$ (blue line). The three results exhibit strong synchronous behaviour $(\bar{R}>0.9)$ for many values of $g_{\mathrm{ex}}$ when $g_{\mathrm{ex}} \gtrsim 0.4 \mathrm{nS}$. However, for $g_{\text {ex }} \lesssim 0.4 \mathrm{nS}$, it is possible to see synchronous behaviour only for $b=70 \mathrm{pA}$ in the range $0.15 \mathrm{nS}<g_{\mathrm{ex}}<0.25 \mathrm{nS}$. The curves have a bimodal nature, the minimal point happening due to the fact of the transition between spiking and bursting synchronisation occurs by means of a process of desynchronisation. In addition, we calculate the coefficient of variation $(\mathrm{CV})$ to determine the range in $g_{\mathrm{ex}}$ where the neurons of the network have spiking or bursting behaviour (Fig. 4(b)). We consider that for $\mathrm{CV}<0.5$ (black dashed line) the neurons exhibit spiking behaviour, while for $\mathrm{CV} \geq 0.5$ the neurons present bursting behaviour. We observe that in the range $0.15 \mathrm{nS}<g_{\mathrm{ex}}<0.25 \mathrm{nS}$ for $b=70 \mathrm{pA}$ there is spiking synchronisation, and bursting synchronisation for $g_{\mathrm{ex}} \gtrsim 0.4 \mathrm{nS}$.

When $g_{\text {ex }}$ is small, our neuronal network is like weakly coupled oscillators (WCO). The theory of WCO can be utilised not only to predict phase locking, but also to reduce the dynamics to a set of phase equations (Hoppensteadt \& Izhikevich, 1997). Hansel et al. (Hansel et al, 1993) studied Hodgkin-Huxley neurons coupled by weak excitatory interactions. They showed that excitatory coupling may have significant consequences in the firing rates between the neurons. In fact, we observe that when aEIF neurons are weakly connected $\left(g_{\mathrm{ex}}<0.1\right)$ by excitatory connections, neurons present significant levels of spiking synchronisation.
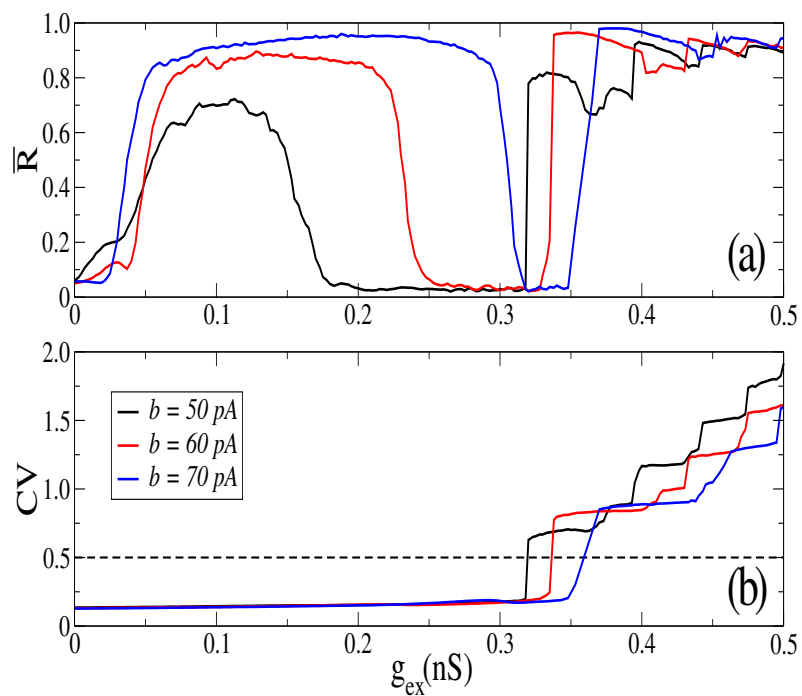

Figure 4: (Colour online) (a) Time-average order parameter and (b) $\mathrm{CV}$ of the excitatory neuronal network for $V_{r}=-58 \mathrm{mV}, p=0.5$, $b=50 \mathrm{pA}$ (black line), $b=60 \mathrm{pA}$ (red line), and $b=70 \mathrm{pA}$ (blue line).

Ostojic (Ostojic, 2014) studied asynchronous activity in balanced networks of excitatory and inhibitory neurons in integrate-and-fire (LIF). We study the influence of the contribution of the relative inhibitory and excitatory strength on the firing patterns. Figures 5(a) and 5(b) exhibit $\bar{R}$ and CV, respectively, for the neuronal network with $80 \%$ of excitatory and $20 \%$ of inhibitory connections. Comparing the results of Fig. 5 for $g_{\text {in }} / g_{\text {ex }}=1$ with Fig. 4 (blue line), we find similar behaviour in both cases, namely spiking and bursting synchronisation with a transition region. However, when $g_{\text {in }} / g_{\text {ex }}=4$ the overall synchronous level, measured by the order parameter (Fig. 5(a)), decreases. Moreover we verify desynchronised spikes for $0<g_{\text {ex }} \lesssim 1.6$, as well as desynchronised spikes and bursts for $g_{\text {ex }} \gtrsim 1.6$ (Fig. $5(\mathrm{~b})$ ). The increase of the relative inhibition strength suppresses the synchronisation.

\section{Parameter space of synchronisation}

The synchronous behaviour depends on the synaptic conductance and the probability of connections. In this 

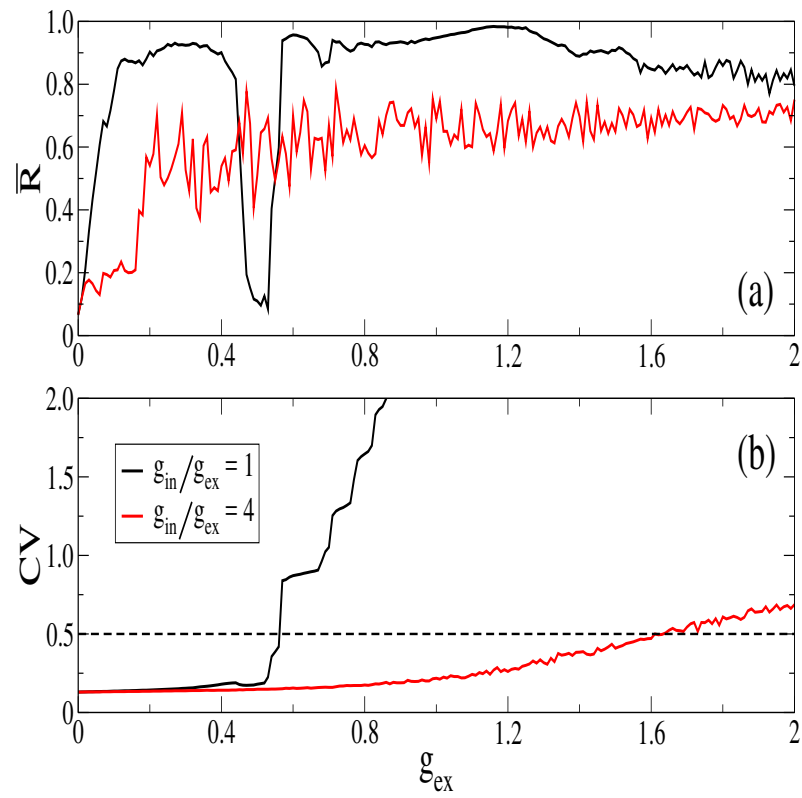

Figure 5: (Colour online) (a) Time-average order parameter and (b) $\mathrm{CV}$ of the excitatory-inhibitory neuronal network for $V_{r}=-58 \mathrm{mV}$, $p=0.5, b=70 \mathrm{pA}, V_{r}=-58 \mathrm{mV}$, and $I_{i}=509.7 \mathrm{pA}$, where we consider $g_{\text {in }} / g_{\text {ex }}=1$ (black line) and $g_{\text {in }} / g_{\text {ex }}=4$ (red line).

Section, we analyse the parameter space of synchronisation of the excitatory neuronal network. Figure 6 exhibits the time-averaged order parameter in colour scale as a function of $g_{\mathrm{ex}}$ and $p$. We verify a large parameter region where spiking and bursting synchronisation is strong, characterised by $\bar{R}>0.9$. The regions I and II correspond to spiking and bursting patterns, respectively, and these regions are separated by a white line with circles. We obtain the regions by means of the coefficient of variation $(\mathrm{CV})$. There is a transition between region I and region II, where neurons initially synchronous in the spike, loose spiking synchronicity to give place to a neuronal network with a regime of bursting synchronisation.

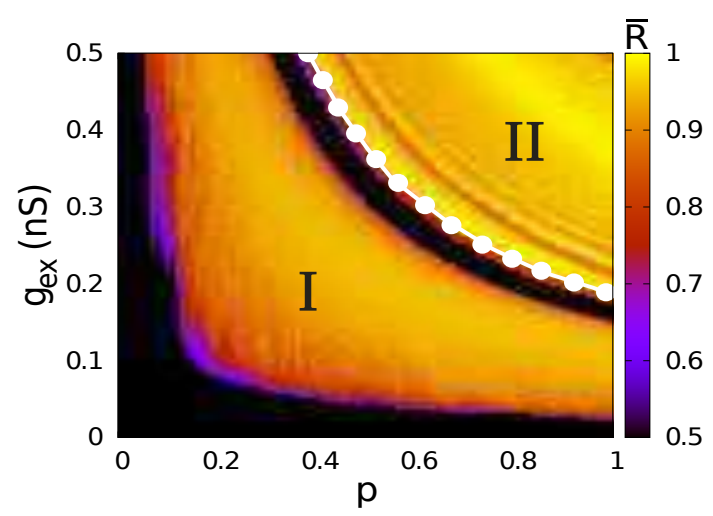

Figure 6: (Colour online) Excitatory synaptic conductance $g_{\mathrm{ex}}$ as a function of the probability $p$ for $V_{r}=-58 \mathrm{mV}$ and $b=70 \mathrm{pA}$, where the colour bar represents the time-average order parameter. The regions I (spike patterns) and II (bursting patterns) are separated by the white line with circles.
We investigate the dependence of spiking and bursting synchronisation on the control parameters $b$ and $V_{r}$. To do that, we use the time average order parameter and the coefficient of variation. Figure 7 shows that the spike patterns region (region I) decreases when $g_{\mathrm{ex}}$ increases. This way, the region I for $b<100 \mathrm{pA}$ and $V_{r}=-49 \mathrm{mV}$ of parameters leading to no synchronous behaviour (Fig. $7(\mathrm{a}))$, becomes a region of parameters that promote synchronised bursting (Fig. 7(b) and 7(c)). However, a large region of desynchronised bursting appears for $g_{\mathrm{ex}}=0.25 \mathrm{nS}$ about $V_{r}=-45 \mathrm{mV}$ and $b>100 \mathrm{pA}$ in the region II (Fig. $7(\mathrm{~b}))$. For $g_{\mathrm{ex}}=0.5 \mathrm{nS}$, we see, in Fig. $7(\mathrm{c})$, three regions of desynchronous behaviour, one in the region I for $b<100 \mathrm{pA}$, other in region II for $b<200 \mathrm{pA}$, and another one is located around the border (white line with circles) between regions I and II for $b>200 \mathrm{pA}$.
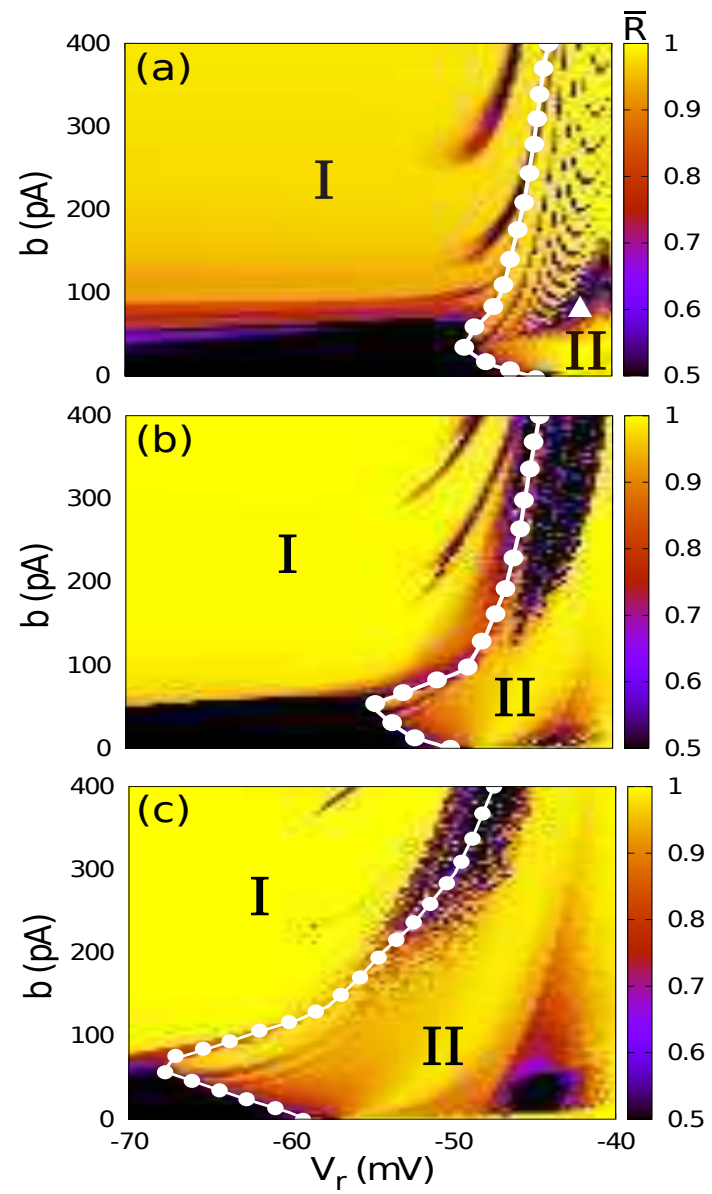

Figure 7: (Colour online) Parameter space $b \times V_{r}$ for $p=0.5, \gamma=0$ (a) $g_{\mathrm{ex}}=0.05 \mathrm{nS}$, (b) $g_{\mathrm{ex}}=0.25 \mathrm{nS}$, and (c) $g_{\mathrm{ex}}=0.5 \mathrm{nS}$, where the colour bar represents the time-average order parameter. The regions I (spike patterns) and II (bursting patterns) are separated by white circles. The white triangle corresponds to $b=86 \mathrm{pA}$ and $V_{r}=-43 \mathrm{mV}$.

Figure 7 shows multiple regions of asynchronous behaviour in II. In this region, we observe the existence of not only bursting behaviour happening for long time intervals, but also happening for short time intervals such as shown in Fig. 8(a). Figs. 8(a) and 8(b) exhibit the action 
potential and the raster plot, respectively, for parameters in the region II shown in Fig. 7(a) with a white triangle. In general, $\mathrm{CV}>0.5$ characterises a network with neurons presenting irregular spikes. More specifically, we have verified in our neuronal network model that $\mathrm{CV}>0.5$ characterise a network with a bursting pattern. The signal, shown in Fig. $8, \bar{R}$ is equal to 0.54 and $\mathrm{CV}$ is equal to 2.96 .
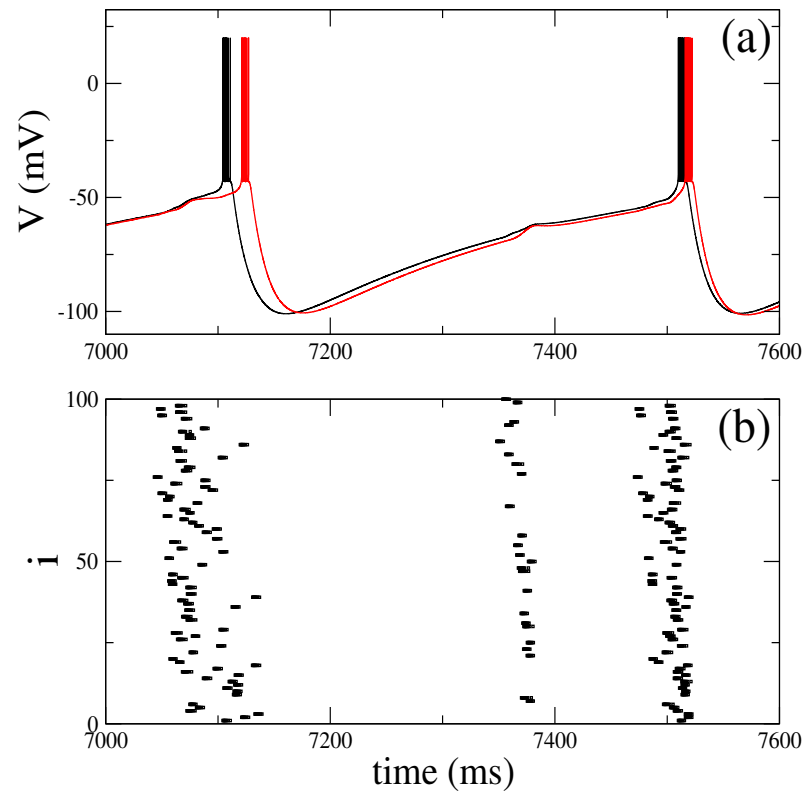

Figure 8: (Colour online) (a) Action potential for two neurons and (b) raster plot for $p=0.5, b=86 \mathrm{pA}, V_{r}=-43 \mathrm{mV}, g_{e x}=0.05 \mathrm{nS}$ and $I_{i}=509.7 \mathrm{pA}$.

It has been found that external perturbations on neuronal networks not only can induce synchronous behaviour (Baptista et al., 2006; Zhang et al., 2015), but also can suppress synchronisation (Lameu et al., 2016). Aiming to study the robustness to perturbations of the synchronous behaviour, we consider an external perturbation $\Gamma_{i}$ in Eq. (3) described by a uniform discrete random process which takes values at each integration step within the interval $[0,1]$. It is applied on each neuron $i$ with an average time interval of about $10 \mathrm{~ms}$ and with a constant intensity $\gamma$ during $1 \mathrm{~ms}$. We also study how this external perturbation disturbs a neuronal network. We initially set an unperturbed neuronal network for parameters that leads to synchronous behaviour. When synchronisation is achieved, we turn the external perturbation on.

Figure 9 shows the plots $g_{\mathrm{ex}} \times p$ for $\gamma>0$, where the regions I and II correspond to spiking and bursting patterns, respectively, separated by white line with circles, and the colour bar indicates the time-average order parameter values. In this Figure, we consider $V_{r}=-58 \mathrm{mV}, b=70 \mathrm{pA}$, (a) $\gamma=250 \mathrm{pA}$, (b) $\gamma=500 \mathrm{pA}$, and (c) $\gamma=1000 \mathrm{pA}$. For $\gamma=250 \mathrm{pA}$ (Fig. 9(a)) the perturbation does not suppress spike synchronisation, whereas for $\gamma=500 \mathrm{pA}$ the synchronisation is completely suppressed in region I (Fig. 9(b)). In Fig. 9(c), we see that increasing further the constant intensity for $\gamma=1000 \mathrm{pA}$, the external perturbation suppresses also bursting synchronisation in region II. Therefore, the synchronous behaviour in region II is more robust to perturbations than in the region $\mathrm{I}$, due to the fact that the region II is in a range with high $g_{\text {ex }}$ and $p$ values, namely strong coupling and high connectivity.

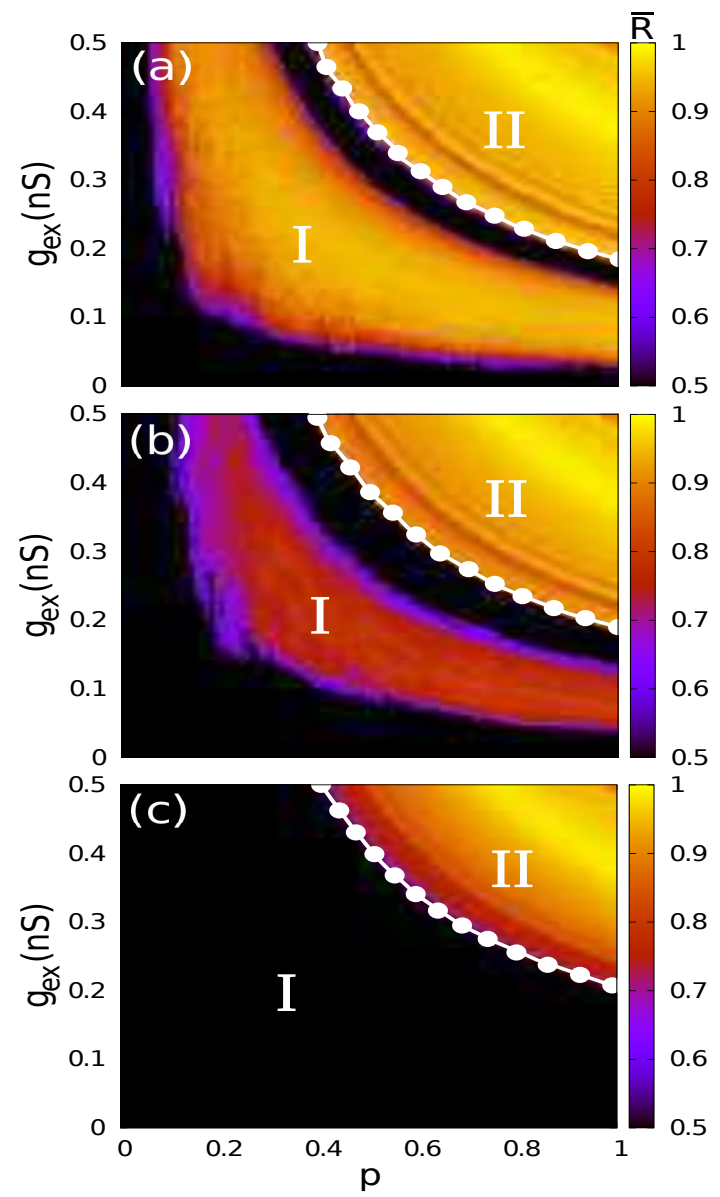

Figure 9: (Colour online) Excitatory synaptic conductance $g_{\mathrm{ex}}$ as a function of the probability $p$ for $V_{r}=-58 \mathrm{mV}, b=70 \mathrm{pA}$, (a) $\gamma=250 \mathrm{pA}$, (b) $\gamma=500 \mathrm{pA}$, and (c) $\gamma=1000 \mathrm{pA}$.

In order to understand the perturbation effect on the spike and bursting patterns, we consider the same values of $g_{\mathrm{ex}}$ and $p$ as Fig. $7(\mathrm{a})$. Figure 10 exhibits the space parameter $b \times V_{r}$, where $\gamma$ is equal to $500 \mathrm{pA}$. The external perturbation suppresses synchronisation in the region I, whereas we observe synchronisation in region II. The synchronous behaviour in region II can be suppressed if the constant intensity $\gamma$ is increased. Therefore, bursting synchronisation is more robust to perturbations than spike synchronisation.

\section{Conclusion}

In this paper, we studied the spiking and bursting synchronous behaviour in a random neuronal network where the local dynamics of the neurons is given by the adaptive exponential integrate-and-fire (aEIF) model. The aEIF 


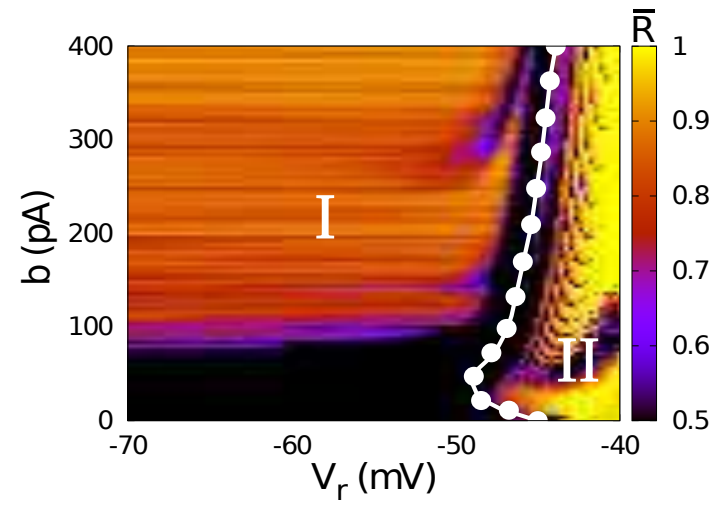

Figure 10: (Colour online) Parameter space $b \times V_{r}$ for $g_{\mathrm{ex}}=0.05 \mathrm{nS}$, $p=0.5$, and $\gamma=500 \mathrm{pA}$, where the colour bar represents the timeaverage order parameter. The regions I (spike patterns) and II (bursting patterns) are separated by white line with circles.

model can exhibit different firing patterns, such as adaptation, tonic spiking, initial burst, regular bursting, and irregular bursting.

In our network, the neurons are randomly connected according to a probability. The larger the probability of connection, and the strength of the synaptic connection, the more likely is to find bursting synchronisation.

It is possible to suppress synchronous behaviour by means of an external perturbation. However, synchronous behaviour with higher values of $g_{\mathrm{ex}}$ and $p$, which typically promotes bursting synchronisation, are more robust to perturbations, then spike synchronous behaviour appearing for smaller values of these parameters. Moreover, we have verified that bursting synchronisation is more robust not only to larger $\gamma$ values, but also for different external perturbation frequencies. The synchronised region in $g_{\text {ex }} \times p$ depends on the external perturbation amplitude and frequency. We concluded that bursting synchronisation provides a good environment to transmit information when neurons are strongly perturbed (large $\Gamma$ ).

The neurons desynchronise near the border between regions I to II. The dynamical fundamentals for this transition to occur are still unclear to us and require further investigation. However, giving the alignment of the border along the vertical coordinates $b$, this phenomenon is critically dependent on the resetting potential $V_{r}$ in that for larger values it induces bursting behaviour.

\section{Acknowledgements}

This study was possible by partial financial support from the following Brazilian government agencies: CNPq (433782/2016-1), CAPES, and FAPESP (2011/19296-1, 2015/07311-7 and 2016/16148-5). We also wish thank Newton Fund and COFAP.

Axmacher, N., Mormann, F., Fernández, G., Elger, C. E. \& Fell, J. (2006). Memory formation by neuronal synchronization. Brain Research Reviews, 52 (1), 170-182.
Badel, L., Lefort, S., Brette, R., Petersen, C. C. H., Gerstner, W. \& Richardson, M. J. E. (2008). Dynamic I-V curves are reliable predictors of naturalistic pyramidal-neuron voltage traces. Journal of Neurophysiology, 99, 656-666.

Batista, C. A. S., Lopes, S. R., Viana, R. L. \& Batista, A. M. (2010). Delayed feedback control of bursting synchronization in a scalefree neuronal network. Neural Networks, 23, 114-124.

Baptista, M. S., Zhou, C. \& Kurths, J. (2006). Information transmission in phase synchronous chaotic arrays. Chinese Physics Letters, 23 (3), 560-563.

Baptista, M. S., Ren, H.-P., Swarts, J. C. M., Carareto, R., Nijmeijer, H. \& Grebogi, C. (2012). Collective Almost Synchronisation in Complex Networks. Plos One, 7, e48118.

Bennet, M., Schatz, M. F., Rockwood, H. \& Wiesenfeld, K. (2002). Huygens's clocks. Proceedings: Mathematical, Physical and Engineering Sciences, 458, 563-579.

Borges, R. R., Borges, F. S., Lameu, E. L., Batista, A. M., Iarosz, K. C., Caldas, I. L., Viana, R. L. \& Sanjuán, M. A. F. (2016). Effects of the spike timing-dependent plasticity on the synchronisation in a random Hodgkin-Huxley neuronal network. Communications in Nonlinear Science \& Numerical Simulation, 34, 12-22.

Brette, R. \& Gerstner, W. (2005). Adaptive exponential integrateand-fire model as an effective description of neuronal activity. Journal of Neurophysiology, 94, 3637-3642.

Clopath, C., Jolivet, R., Rauch, A., Lüscher, H.-R. \& Gerstner, W. (2007). Predicting neuronal activity with simple models of the threshold type: Adaptive Exponential Integrate-and-Fire model with two compartments. Neurocomputing, 70 (10-12), 1668-1673.

Davison, A. P., Feng, J. \& Brown, D. (2001). Spike synchronization in a biophysically-detailed model of the olfactory bulb. Neurocomputing, 38-40, 515-521.

Erdös, P. \& Rényi, A. (1959). On random graphs, I. Publicationes Mathematicae, 6, 290-297.

Fell, J., \& Axmacher, N. (2011). The role of phase synchronization in memory processes. Nature Reviews Neuroscience, 12, 105-118.

Gabbiani, F., \& Koch, C. (1998). Principles of spike train analysis, in Methods in Neuronal Modeling: From Ions to Networks, 2nd Edn, eds C. Koch and I. Segev (Cambridge, MA: MIT Press), 313-360.

Gelenbe, E., \& Cramer, C. (1998). Oscillatory corticothalamic response to somatosensory input ${ }^{1}$. Biosystems, 48 (1-3), 67-75.

Hansel, D., Mato, G. \& Meunier, C. (1993). Phase dynamics for weakly coupled Hodgkin-Huxley neurons. Europhysics Letters 23 (5), 367-370.

Hindmarsh, L. J., \& Rose, R. M. (1984). A model of neuronal bursting using three coupled first order differential equations. Proceedings of the Royal Society of London Series B, 221 (1222), 87-102.

Hipp, J. F., Engel, A. K., \& Siegel, M. (2011). Oscillatory synchronization in large-scale cortical networks predicts perception. Neuron, 69 (2), 387-396.

Hodgkin, A. L., \& Huxley, A. F. (1952). A quantitative description of membrane current and its application to conduction and excitation in nerve. The Journal of Physiology, 117 (4), 500-544.

Hoppensteadt, F. C. \& Izhikevich, E. M. (1997). Weakly connected neural networks. New York: Springer.

Huang, J., Li, C., Huang, T., \& He, X. (2014). Finite-time lag synchronization of delayed neural networks. Neurocomputing, 139, 145-149.

Hutcheon, B., \& Yarom, Y. (2000). Resonance oscillation and the intrinsic frequency preferences of neurons. Trends In Neuroscience, 23 (5), 216-222.

Jefferys, J. G. R., \& Haas, H. L. (1982). Synchronized bursting of CA1 hippocampal pyramidal cells in the absence of synaptic transmission. Nature, 300, 448-450.

Kuramoto, Y. (2003). Chemical oscillations, waves and turbulence. New York: Dover Publications.

Lameu, E. L., Borges, F. S., Borges, R. R., Iarosz, K. C., Caldas, I. L., Batista, A. M., Viana, R. L. \& Kurths, J. (2016). Suppression of phase synchronisation in network based on cat's brains. Chaos, $26,043107$.

de Lange, E. \& Hasler, M. (2008). Predicting single spikes and spike 
patterns with the Hindmarsh-Rose model. Biological Cybernetics, 99, 349-360.

Lapicque, L. (1907). Recherches quantitatives sur l'excitation électrique des nerfs traitée comme une polarization. Journal de Physiologie et de Pathologie Générale, 9, 620-635.

Li, Y., \& Li, C. (2016). Complete synchronization of delayed chaotic neural networks by intermittent control with two switches in a control period. Neurocomputing, 173 (3), 1341-1347.

Maeda, E., Robinson, H. P. C., \& Kawana, A. (1995). The mechanisms of generation and propagation of synchronized bursting in developing networks of cortical neurons. The Journal of Neuroscience, 15 (10), 6834-6845.

Naud, R., Marcille, N., Clopath, C., \& Gerstner, W. (2008). Firing patterns in the adaptive exponential integrate-and-fire model. Biological Cybernetics, 99, 335-347.

Ostojic, S. (2014). Two types of asynchronous activity in networks of excitatory and inhibitory spiking neurons. Nature Neuroscience, 17 (4), 594-600.

Pereira, T., Baptista, M. S., \& Kurths, J. (2007). Onset of phase synchronisation in neurons with chemical synapse. International Journal of Bifurcation and Chaos, 17 (10), 3545-3549.

Pikovsky, A., Rosenblum, M., \& Kurths, J. (2001). Synchronization: A universal concept in nonlinear sciences. Cambridge: Cambridge University Press.

Riehle, A., Grün, S., Diesmann, M., \& Aertsen, A. (1997). Spike synchronization and rate modulation differentially involved in motor cortical function. Science, 278, 1950-1953.

Rulkov, N. F. (2001). Regularization of synchronized chaotic bursts. Physical Review Letters, 86 (1), 183-186.

Senn, W., Wyler, K., Streit, J., Larkum, M., Lüscher H.-R., Mey, H., Müller, L., Stainhauser, D., Vogt, K., \& Wannier, Th. (1996). Dynamics of a random neural network with synaptic depression. Neural Networks, 9 (4), 575-588.

Steinmetz, P. N., Roy, A., Fitzgerald, P. J., Hsiao, S. S., Johnson, K. O., \& Niebur, E. (2000). Attention modulates synchronized neuronal firing in primate somatosensory cortex. Nature, 404, 187190.

Touboul, J. \& Brette, R. (2008). Dynamics and bifurcations of the adaptive exponential integrate-and-fire model. Biological Cybernetics, 99, 319-334.

Viana, R. L., Borges, F. S., Iarosz, K. C., Batista, A. M., Lopes, S. R., \& Caldas, I. L. (2014). Dynamic range in a neuron network with electrical and chemical synapses. Communications in Nonlinear Science and Numerical Simulation, 19, 164-172.

Zhang, W., Li, C., Huang, T., \& Xiao, M. (2015). Synchronization of neural networks with stochastic perturbation via aperiodically intermittent control. Neural Networks, 71, 105-111.

Wang, X.-J. (2010). Neurophysiological and Computational Principles of Cortical Rhythms in Cognition. Physiological Reviews, 90 (3), 1195-1268.

Wu, Y., Lu, W., Lin, W., Leng, G., \& Feng, J. (2012). Bifurcations of emergent bursting in a neuronal network. Plos One, 7 (6), e38402. 